\title{
Leak Profile Inspection During Nasal Continuous Positive Airway Pressure
}

\author{
Marcel A Baltzan MD DABSM, Richard Dabrusin MD FRCPC, Alfonso Garcia-Asensi RPGST, \\ Jennie-Laure Sully MSc, Maryse Parenteau RPGST, Germaine Tansimat RN, \\ Ibrahim Kassissia MD, and Norman Wolkove MD FCCP
}

\begin{abstract}
BACKGROUND: Patients treated with nasal continuous positive airway pressure (nasal CPAP) for obstructive sleep apnea (OSA) often have adverse effects from and poor adherence to CPAP. OBJECTIVE: To describe abnormal CPAP leak profiles and assess inter-observer reliability in identifying leak profiles and the correlation of leak profiles with leak rate and clinical outcomes. METHODS: In a sleep-disorders clinic we prospectively studied 35 consecutive patients newly diagnosed with moderate or severe OSA, and who had undergone polysomnographic diagnosis and nasal CPAP titration. We analyzed the data recorded by their CPAP machines during their first week of CPAP. Two independent clinical sleep specialists inspected each night's leak profiles. We defined a "continuous" leak profile segment as a leak increase of $\geq 20 \mathrm{~L} / \mathrm{min}$ for $>5 \mathrm{~min}$. We defined a "serrated" leak profile segment as a leak that oscillated up to $\geq 20 \mathrm{~L} / \mathrm{min}$ in $\leq 5 \mathrm{~min}$. With a validated questionnaire, we surveyed the patients about adverse effects. RESULTS: Overall inter-observer agreement was $\mathbf{8 8 \%}$ for continuous leak and $\mathbf{9 2 \%}$ for serrated leaks. The kappa values were 0.76 and 0.85 , respectively. Deviance $( \pm 2$ SD) between scorers was $-14 \%$ to $11 \%$ for continuous leaks, and $-15 \%$ to $9 \%$ for serrated leaks. The duration of manually scored profiles correlated modestly but significantly with the machine-recorded leaks. The mean \pm SD adherence to CPAP was lower in the patients with the highest quartile of continuous leak $(5.28 \pm 2.24 \mathrm{~h} / \mathrm{night}$ versus $6.66 \pm 1.72 \mathrm{~h} / \mathrm{night})$. Adverse effects increased with increasing serrated leak $(P=.01)$. CONCLUSIONS: Manually scored leak profiles in patients treated with nasal CPAP can guide clinicians with respect to short-term adherence to nasal CPAP and adverse effects. Key words: sleep apnea; sleep apnea syndromes; polysomnography; continuous positive airway pressure; CPAP. [Respir Care 2011;56(5):591-595. (C) 2011 Daedalus Enterprises]
\end{abstract}

\section{Introduction}

The most commonly used therapy for obstructive sleep apnea (OSA) is continuous positive airway pressure

Marcel A Baltzan MD DABSM, Richard Dabrusin MD FRCPC, JennieLaure Sully MSc, and Norman Wolkove MD FCCP are affiliated with Mount Sinai Hospital Center, Montréal, Québec, Canada. Marcel A Baltzan MD DABSM, Alfonso Garcia-Asensi RPGST, Maryse Parenteau RPGST, Germaine Tansimat RN, and Ibrahim Kassissia MD are affiliated with OSR Médical Sleep Disorders Center, Montréal, Québec, Canada. Ms Sully is also affiliated with Université de Montréal, Montréal, Québec, Canada.

This research was partly supported by the Mount Sinai Hospital Research Foundation and the OSR Médical Sleep Disorders Center.

The authors have disclosed no conflicts of interest.
(CPAP). ${ }^{1-4}$ This is usually administered via nasal mask (nasal CPAP), which is perceived as more comfortable than oronasal mask. ${ }^{5}$ Adverse effects are common, ${ }^{6-10}$ and overall adherence to CPAP therapy is poor, because patients often abandon therapy within the first month. ${ }^{11-14}$

\footnotetext{
Marcel A Baltzan MD DABSM presented a version of this paper at the 104th International Conference of the American Thoracic Society, held May 16-21, 2008, in Toronto, Ontario, Canada.

Correspondence: Marcel A Baltzan MD DABSM, Mount Sinai Hospital Center, 5690 Cavendish Boulevard, Cote St-Luc (Montréal) Québec, Canada H4W 1S7. E-mail: marc.baltzan@clinepi.mcgill.ca.
}

DOI: $10.4187 /$ respcare.00977 
The monitoring of CPAP therapy is now possible with the advent of CPAP machines that gather statistical and graphical information on use and efficacy. Current CPAP machines collect data on time-at-pressure, apneas, hypopneas, snoring, and leaks, and those data are important in clinical follow-up, especially if the patient initiated on auto-titrating CPAP, without polysomnographic titration. ${ }^{15}$ Current CPAP machines can also create graphic profiles that describe the data. However, the utility of this wealth of information is unclear. Studies to correlate the CPAP machine data with adverse effects and adherence to therapy are lacking. Specifically, it has not been established that the data collected provides information that allows clinicians to modify CPAP. The use of such information shows promise in improving patient care. ${ }^{16}$

SeE the Related Editorial on Page 718

Based on our clinical observations, we postulated that OSA patients on nasal CPAP have discernible leak patterns that can be reliably interpreted. These leak patterns may reflect phenomena that impact patient adverse effects, adherence to therapy, and patient satisfaction with nasal CPAP. To test this hypothesis we first explored a group of consecutive subjects who were starting nasal CPAP treatment. Our primary goal was to describe and evaluate 2 types of leak patterns. We studied inter-scorer concordance in profile interpretation, and correlated machine-recorded leaks with the duration of the profile ratings. To explore the potential meaning of these profiles, we also administered a questionnaire to patients to determine whether patient satisfaction and adverse effects were related to these pressure profiles. We also studied the relationship of the leak profiles to CPAP adherence.

\section{Methods}

\section{Study Design}

We prospectively studied consecutive newly diagnosed patients who were prescribed single-pressure nasal CPAP. All the patients were recruited from the specialized outpatient sleep clinic of Mount Sinai Hospital Center, Montreal, Québec, Canada. The study was approved by the Mount Sinai Hospital ethics committee, and we followed the ethical standards prescribed by Québec law and the Declaration of Helsinki.

A group of 35 consecutive patients qualified out of a consecutive recruitment of 79 patients. Among those not included were 9 whose CPAP was titrated with an oronasal mask; 10 men who had upper-lip facial hair (moustaches), which is expected to cause leak at the mask interface; 23 patients who were not committed to using CPAP; and 12 who declined consent for personal reasons, including time and travel constraints.
Inclusion in the study required verification of the OSA diagnosis, with an apnea-hypopnea index of $>15$ events per hour, via polysomnography. If central apneas were scored, more obstructive rather than central apneas were required to be present, with a central apnea index of less than 5 apneas per hour. We excluded patients with severe cardio-respiratory or neurologic disease, variable-pressure therapy, or supplemental oxygen. Patients were evaluated for the study after their first week on nasal CPAP at home, and up to 7 more times over their first 3 months of nasal CPAP, for education and mask fitting, and were offered a change of nasal mask if they sensed the fit was not adequate. They also underwent weekly monitoring of leak, event, and adherence during the first 4 weeks and at the end of 3 months. The data used for the present analyses were from the data download after the first week of nasal CPAP. No external measure of leak was added to the protocol, as we intended to target the clinical population with minimal experimental instrumentation, not to measure the nasal CPAP leak per se, but to analyze the profiles for potentially clinically relevant patterns.

\section{Leak Profile Scoring}

Based on clinical experience, 2 distinct leak patterns were selected. A "continuous" leak profile segment was defined as a rapid leak increase of $\geq 20 \mathrm{~L} / \mathrm{min}$, above baseline leak, that was sustained for $\geq 5 \mathrm{~min}$. We defined a "serrated" leak profile segment as a leak that oscillated up to $\geq 20 \mathrm{~L} / \mathrm{min}$ in $\leq 5 \mathrm{~min}$. We observed that the absolute level of leak with a properly applied mask depends on the mask whisper valve characteristics, the CPAP pressure, and perhaps other mechanical mask characteristics. As a result, we did not seek to score the absolute leak values, but instead the relative leak changes during the unsupervised home use periods. We chose the $20 \mathrm{~L} / \mathrm{min}$ cut-off because it was a clearly discernible change on the tracings, and was found to be a threshold that may require attention during positive airway titration polysomnography.

Two independent clinical sleep specialists reviewed the leak profiles by independent inspection of each night's recordings after the first week of treatment. They scored the tracings via visual inspection, based on the a priori defined and written criteria. Each night was scored independently. For each night both scorers determined the percentage of time in the 2 patterns, which was immediately recorded as a percentage on a separate scoring sheet for later inter-scorer comparison. The scorers were blinded to each other's scoring. Scores were deemed to agree if they were within $10 \%$. For correlation with machine leak statistics, adverse effects, and adherence, average leak pattern percentages were calculated for each patient. A night was disqualified if less than 2 hours were recorded. 


\section{Measurements}

We evaluated several CPAP machines for possible use in this study, and chose the REMStar (Respironics, Murrysville, Pennsylvania) because it provided the longest period of full data collection (7 days of graphic leak profiles) and several leak and adherence statistics.

We investigated the relationship of the leak profiles with adverse effects and satisfaction with CPAP. The adverse effects questionnaire we used has been validated. ${ }^{10}$ The questions are on a 7-point ordinal scale, and the respondent selfrates the frequency of morning headache, morning dry mouth, nasal congestion, rhinorrhea, and air leak out the mouth. Overall satisfaction with CPAP treatment is rated on a zero (not at all satisfied) to 10 (extremely well satisfied) scale.

Nocturnal diagnostic polysomnography had been performed on all the patients. Sleep staging was performed with the standard polysomnographic montage $(\mathrm{C} 3, \mathrm{C} 4$, $\mathrm{O} 1, \mathrm{O} 2$, electrooculogram, and electroencephalogram). Additional measurements included air flow (measured via nasal pressure transducer and thermistor), thoraco-abdominal movements (measured via inductive plethysmography), and pulse oximetry. Registered polysomnographic technologists scored the studies, using standard criteria. ${ }^{17-19}$

\section{Statistical Analysis}

The primary analysis of inter-scorer agreement was calculated on a per-night basis for each of the leak profiles. We calculated the kappa values, percent agreement, and deviance between scorers. ${ }^{20} \mathrm{We}$ also calculated the correlations between the week's leak profile averages and the leak statistics generated by the CPAP machine, which also compiled machine-measured variables over the data-collection week. We initially analyzed the adverse effects, CPAP adherence, and patient satisfaction by graphing in quartiles for visual inspection of linearity, prior to statistical analysis. We performed statistical tests of associations with a $t$ test for continuous variables. Statistical significance was declared at a 2 -sided $P<.05$. For the multiple planned comparisons of both manually scored leak profiles with overall adverse effects, adherence, and satisfaction, we made a Bonferroni adjustment to state significance with a significant $P<.008$. A power analysis estimated that at least 31 patients with a specific leak profile were required to detect a one-hour reduction in adherence to a beta error of 0.80 . A post-hoc calculation revealed that the final power of the study was over 0.95 .

\section{Results}

Table 1 describes the patients. Leak profiles could clearly be scored on the tracings (Fig. 1). Table 2 shows the machine-measured variables and the manually scored leak times.
Table 1. Subjects

\begin{tabular}{lc}
\hline \hline Age, mean (range), y & $53(33-78)$ \\
Male, no. (\%) & $24(71)$ \\
Body mass index, mean (range), $\mathrm{kg} / \mathrm{m}^{2}$ & $31.6(22.7-48.8)$ \\
Epworth sleepiness score (range 0-24), & $13(5-24)$ \\
$\quad$ mean (range) & $9(5-13)$ \\
CPAP, mean (range), cm $\mathrm{H}_{2} \mathrm{O}$ & $6: 20(0: 51-9: 33)$ \\
Compliance, mean (range), h, all nights
\end{tabular}
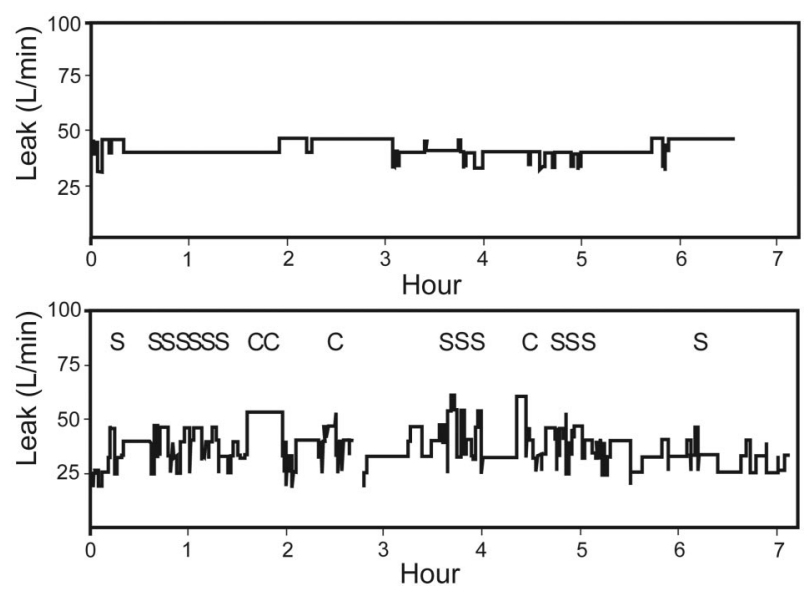

Fig. 1. Examples of leak profile scoring. Upper panel: continuous leak $0 \%$ of the time; serrated leak $0 \%$ of the time. Lower panel: continuous leak (at points labeled C) $7 \%$ of the time; serrated leak (at points labeled S) $27 \%$ of the time.

Table 2. Leak Characteristics During the First Week of Nasal CPAP

\begin{tabular}{lc}
\hline \hline & Mean (range) \\
\hline Average leak (L/min) & $42(32-54)$ \\
Average maximum leak (L/min) & $84(62-108)$ \\
Average 90\% leak (L/min) & $52(38-67)$ \\
Time with large leak $(\%)$ & $1(0-13)$ \\
Time with continuous leak $(\%)$ & $9(0-40)$ \\
Time with serrated leak $(\%)$ & $18(0-62)$ \\
\hline
\end{tabular}

Overall inter-scorer agreement was $88 \%$ for continuous leak (kappa 0.76) and 92\% for serrated leaks (kappa 0.85). The ranges $( \pm 2 \mathrm{SD}$ ) of inter-scorer deviance were $-14 \%$ to $11 \%$ for continuous leak, and $-15 \%$ to $9 \%$ for serrated leak.

Table 3 shows the correlations between the manually scored leaks and the machine-recorded leaks. Both the continuous and serrated manually scored leaks correlated moderately with the overall average leak and the average $90 \%$ leak.

Adverse effects, as a whole, increased with increasing serrated leak. Adverse effects were more frequent and more severe with increasing serrated leak $(P<.001)$ (Fig. 2). The adverse effect oral air leak also correlated with serrated leak $(P=.007)$. Adverse effects did not correlate with continuous leak $(P=.14)$.

Adherence did not correlate with serrated leaks $(P=.91)$. The quartile with the higher continuous leak times suggested 
Table 3. Correlation of CPAP-Machine-Recorded Leak and Manually Scored Leak

\begin{tabular}{lll}
\hline \hline & \multicolumn{2}{c}{ Correlation Coefficient $(P)$} \\
\cline { 2 - 3 } & \multicolumn{1}{c}{ Continuous } & Serrated \\
\hline Average large leak \% (all nights) & $0.50(.002)$ & $0.23(.20)$ \\
Average maximum leak & $0.24(.18)$ & $0.34(.052)$ \\
Average 90\% leak & $0.64(<.001)$ & $0.50(.002)$ \\
Average leak & $0.54(<.001)$ & $0.43(.01)$ \\
\hline
\end{tabular}

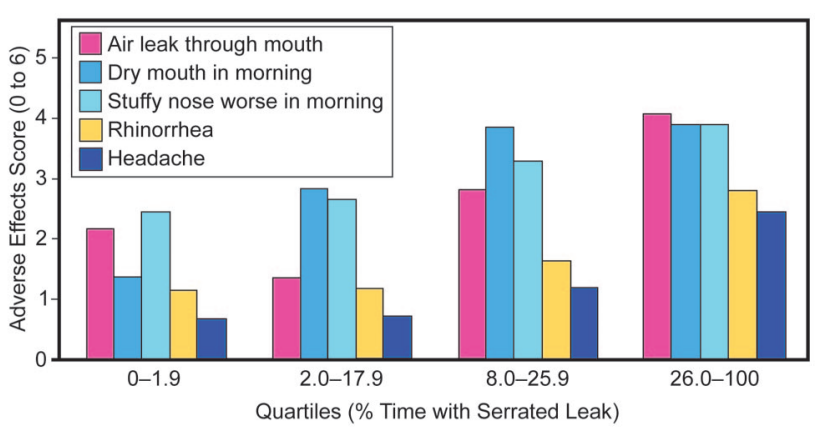

Fig. 2. Adverse effects of nasal CPAP relative to the quartiles of percent of time with serrated leak profile. There is an increasing frequency of adverse effects with increasing serrated leak time $(P<.001)$.

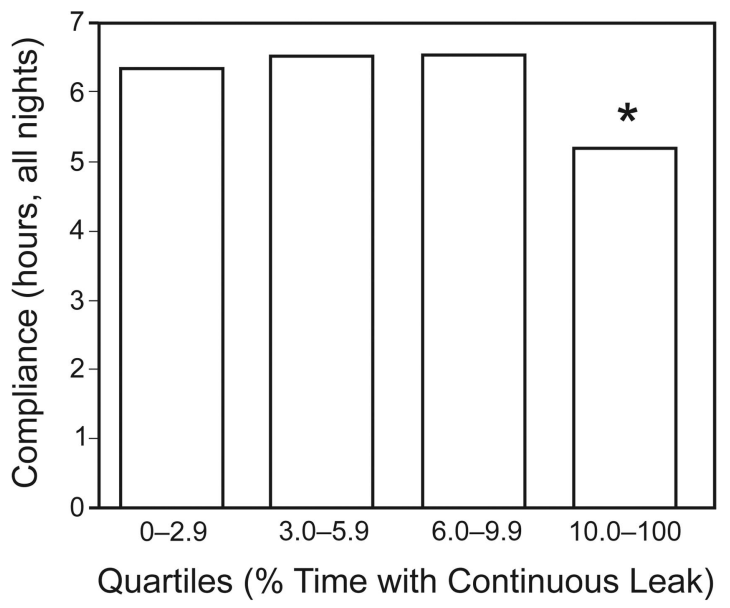

Fig. 3. Nasal CPAP adherence with respect to the quartiles of percent of time with continuous leak. ${ }^{*} P=.07$.

lower adherence to nasal CPAP (Fig. 3), but that difference did not remain significant after Bonferroni correction.

Satisfaction did not correlate with the serrated leak or continuous leak scores $(P=.06$ and .13 , respectively). Patients who sensed leak around the nasal mask and required at least one change of mask type for better fit had, on average, higher continuous leak scores $(8.90 \mathrm{SD}, 11.0 \%$ of time) than those who did not require a change of mask (5.74 SD, $8.29 \%$ of time), but that difference did not remain significant after Bonferroni correction.

\section{Discussion}

We have shown that the leak profile tracings of patients on nasal CPAP for moderate or severe OSA demonstrate 2 distinct and common patterns we call continuous leak and serrated leak. The leak profile tracings can be reliably scored with explicit criteria, with good inter-scorer agreement. The manually scored leak profile durations correlated modestly with the machine-measured leaks. Adverse effects were higher in patients with more serrated leaks, and the patients with the highest continuous leaks had the worst short-term CPAP adherence.

The majority of patients who are prescribed CPAP for OSA tolerate this therapy and respond well, while others remain on treatment despite adverse effects. ${ }^{5-10}$ The adequacy and comfort of mask fit, the appropriate pressure, and the management of adverse effects strongly influence efficacy. ${ }^{2,4,21} \mathrm{We}$ believe that any immediately available information that helps improve patient outcomes is valuable. The leak profiles give one clue to difficulties a patient may have with nasal CPAP in their first week. We found that serrated leak is more frequent in patients with greater adverse effects, especially air leak from the mouth. Our finding of a possible relationship between the continuous leak profile and adherence to therapy requires further research, as our statistical analysis may have highlighted a chance finding.

\section{Limitations}

We studied only a small number of patients, but this clinical population was carefully selected to be adherent to the protocol to study the leak profiles, including the reliability of visual inspection of the profiles and clinical correlates of these manually scored leak profiles. As a result, the leak frequencies and adverse effects cannot be assumed to be generalizable to all OSA patients. Yet our finding of good inter-scorer agreement and significant correlation with machine-recorded leak statistics supports the internal consistency of this study in distinguishing distinct leak profiles. The next research step would be to monitor patients with attended polysomnography to discern which leak sources resulted in leak profile changes.

Leaks are common in CPAP. Recent guidelines state that these leaks should be minimized with careful mask selection prior to auto-titrating CPAP or manual laboratory titration..$^{15,22}$ Yet in practice, leaks are very common in patients who sleep with CPAP. The leaks may be due to mask fit problems or, with nasal CPAP, mouth leaks, which have been polysomnographically documented to occur more often in patients with frequent mouth breathing during sleep. ${ }^{23}$ This phenomenon correlates with worse adherence to nasal CPAP. ${ }^{23}$ Large leaks can compromise CPAP's effectiveness in treating OSA. When the CPAP machine reads breath-by-breath signals to either trigger ventilatory assis- 
tance or modify the pressure at expiration, leak can render these "intelligent" or "triggered" algorithms ineffective and even disrupt sleep. ${ }^{24}$ We have also found that mouth leak is related to nasal congestion, removal of the nasal CPAP mask, and worse nasal CPAP adherence. ${ }^{10}$

\section{Conclusions}

There are 2 discernible and definable leak patterns in nasal CPAP, serrated leak and continuous leak, which are identifiable with acceptable inter-scorer concordance. There appeared to be a modest correlation of leak pattern duration and overall leak statistics from the CPAP generator. Serrated leak appeared to have a relationship with overall adverse effects and perhaps with adherence in the highest quartile of continuous leak profile. There was a need to change masks more often in patients with more continuous leaks.

\section{REFERENCES}

1. Sullivan CE, Issa FG, Berthon-Jones M, Eves L. Reversal of obstructive sleep apnoea by continuous positive airway pressure applied through the nares. Lancet 1981;1(8225):862-865.

2. Beninati W, Sanders MH. Optimal continuous positive airway pressure for the treatment of obstructive sleep apnea/hypopnea. Sleep Med Rev 2001;5(1):7-23.

3. Patel SR, White DP, Malhotra A, Stanchina ML, Ayas NT. Continuous positive airway pressure therapy for treating sleepiness in a diverse population with obstructive sleep apnea: results of a metaanalysis. Arch Intern Med 2003;163(5):565-571.

4. Gay P, Weaver T, Loube D, Iber C; Positive Airway Pressure Task Force; Standards of Practice Committee; American Academy of Sleep Medicine. Evaluation of positive airway pressure treatment for sleep related breathing disorders in adults. Sleep 2006;29(3):381-401.

5. Mortimore IL, Whittle AT, Douglas NJ. Comparison of nose and face mask CPAP therapy for sleep apnoea. Thorax 1998;53(4):290-292.

6. Hoffstein V, Viner S, Mateika S, Conway J. Treatment of obstructive sleep apnea with nasal continuous positive airway pressure. Patient compliance, perception of benefits, and side effects. Am Rev Respir Dis 1992;145(4 Pt 1):841-845.

7. Pépin JL, Leger P, Veale D, Langevin B, Robert D, Lévy P. Side effects of nasal continuous positive airway pressure in sleep apnea syndrome: study of 193 patients in two French sleep centers. Chest 1995;107(2):375-381.

8. Mador MJ, Krauza M, Pervez A, Pierce D, Braun M. Effect of heated humidification on compliance and quality of life in patients with sleep apnea using nasal continuous positive airway pressure. Chest 2005;128(4):2151-2158.

9. Kalan A, Kenyon GS, Seemungal TAR, Wedzicha JA. Adverse effects of nasal continuous positive airway pressure therapy in sleep apnoea syndrome. J Laryngol Otol 1999;113(10):888-892.
10. Baltzan M, Elkholi O, Wolkove N. Evidence of interrelated side effects with reduced compliance in patients treated with nasal continuous positive airway pressure. Sleep Med 2009;10(2):198-205.

11. Weaver T, Grunstein RR. Adherence to continuous positive airway pressure therapy: the challenge to effective treatment. Proc Amer Thorac Soc 2008;5(2):173-178.

12. Haniffa M, Lasserson TJ, Smith I. Interventions to improve compliance with continuous positive airway pressure for obstructive sleep apnoea. Cochrane Database Syst Rev 2009;(4):CD003531.

13. Engleman H, Wild M. Improving CPAP use by patients with sleep apnoea/hypopnea syndrome (SAHS). Sleep Med Rev 2003;7(1):81-99.

14. Wolkove N, Baltzan MA, Elkholi O, Palayew M. Long-term compliance with continuous positive airway pressure in patients with obstructive sleep apnea. Can Respir J 2008;15(7):365-369.

15. Morgenthaler TI, Aurora RN, Brown T, Zak R, Alessi C, Boehlecke B, et al; Standards of Practice Committee of the AASM. Practice parameters for the use of autotitrating continuous positive airway pressure devices for titrating pressures and treating adult patients with obstructive sleep apnea syndrome: an update for 2007 Sleep 2008;31(1):141-147.

16. Stepnowsky CJ Jr, Palau JJ, Marler MR, Gifford AL. Pilot randomized trial of the effect of wireless telemonitoring on compliance and treatment efficacy in obstructive sleep apnea. J Med Internet Res 2007;9(2): e14. http://www.jmir.org/2007/2/e14. Accessed March 8, 2011.

17. Rechtschaffen A, Kales A, eds. A manual of standardized terminology, techniques, and scoring system for sleep stages of human subjects. Washington, DC: National Institute of Health, Publication 204, US Government Printing Office; 1968.

18. Bonnet m, Carley D, Caskardon M, Easton P, Guilleminault C, Harper $\mathrm{R}$, et al; ASDA (American Sleep Disorders Association) Sleep Disorders Task Force. EEG arousals: scoring rules and examples. Sleep 1992;15(2):173-184.

19. Flemons WW, Buysse D, Redline S, Pack A, Strohl K, Wheatley J; American Academy of Sleep Medicine Task Force. Sleep-related breathing disorders in adults: recommendations for syndrome definition and measurement techniques in clinical research. Sleep 1999; 22(5):667-689.

20. Berry CC. The kappa statistic. JAMA 1992;268(18):2513-2514.

21. Kushida CA, Chediak A, Berry RB, Brown LK, Gozal D, Iber C, et al; Positive Airway Pressure Titration Task Force of the American Academy of Sleep Medicine. Clinical guidelines for the manual titration of positive airway pressure in patients with obstructive sleep apnea. J Clin Sleep Med 2008;4(2):157-171.

21. McNicholas WT. Follow-up and outcomes of nasal CPAP therapy in patients with sleep apnea syndrome. Monaldi Arch Chest Dis 2001; 56(6):535-539.

22. Bachour A, Maasilta P. Mouth breathing compromises adherence to nasal continuous positive airway pressure therapy. Chest 2004;126(4): 1248-1256.

23. Teschler H, Stampa J, Ragette R, Konietzko N, Berthon-Jones M. Effect of mouth leak on effectiveness of nasal bilevel ventilatory assistance and sleep architecture. Eur Respir J 1999;14(6):1251-1257.

24. Guo YF, Sforza E, Janssens JP. Respiratory patterns during sleep in obesity-hypoventilation patients treated with nocturnal pressure support. Chest 2007;131(4):1090-1099.

This article is approved for Continuing Respiratory Care Education credit. For information and to obtain your CRCE

(free to AARC members) visit www.RCJournal.com

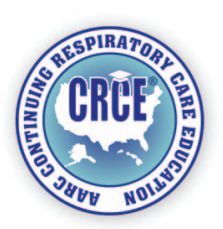

